\title{
Free States and Automorphisms of the Clifford Algebra
}

\author{
J. C. Wolfe` \\ Universiteit Leuven, Leuven, Belgium $\star \star$
}

\begin{abstract}
We study automorphisms of the Clifford algebra which map the set of quasi-free states onto itself. We show that they are quasi-free if the oneparticle space is infinite dimensional, and give counter examples in finite dimensions.
\end{abstract}

In a recent paper [1], Hugenholtz and Kadison have shown an automorphism of the $C A R$ or Clifford algebra which maps the set of gauge invariant quasi-free states onto itself to be quasi-free. The same result is known for automorphisms which preserve the set of all quasi-free states [2]. We give simple, alterative proofs of these two results when the one particle space is infinite dimensional and counterexamples when it is not. Because of its economical description of non-gauge invariant free states and of non-unitary Bogoliubov transformations, we have worked in the real Hilbert space formalism of [3]. The connection between this and the complex Hilbert space formalism used in [1] is found in Section 2 of [3].

Let $(H,()$,$) be a real Hilbert space of even or infinite dimension. The C^{*}$ Clifford algebra $\mathfrak{A}(H)$ over $H$ is generated by the range of a linear map $f \rightarrow B(f)$ of $H$ into self-adjoint part of $\mathfrak{U}(H)$, satisfying

$$
B(f) B(g)+B(g) B(f)=2(f, g) .
$$

If $H^{\prime}$ is a subspace of even or infinite dimension we denote by $\mathfrak{A}\left(H^{\prime}\right)$ the $C^{*}$ subalgebra of $\mathfrak{A}(H)$ generated by $\left\{B(f) \mid f \in H^{\prime}\right\}$. Every orthogonal transformation $\mathcal{O}$ on $H$ defines a $*$-automorphism $\alpha_{\mathfrak{O}}$ of $\mathfrak{A}(H)$ such that

$$
\alpha_{\mathscr{O}} B(f)=B(\mathcal{O} f) \text {. }
$$

Such an automorphism is called quasi-free.

Every anti-hermitian operator $A$ in the unit ball of $B(H)$ defines [3] a state $\omega_{A}$ such that

$$
\omega_{A}\left(B\left(f_{1}\right) \ldots B\left(f_{N}\right)\right)=\left\{\begin{array}{l}
0 \text { if } N \text { is odd } \\
\sum_{i=2}^{N}(-1)^{i} \omega_{A}\left(B\left(f_{1}\right) B\left(f_{i}\right)\right) \omega_{A}\left(B\left(f_{2}\right) \ldots \widehat{\left.B\left(f_{i}\right) . . B\left(f_{N}\right)\right)}\right.
\end{array}\right.
$$

otherwise

* Derde Cyclus Fellow, K.U.L. Belgium.

$\star \star$ Postal address: Physics Department, University of British Columbia, Vancouver, B.C., Canada. 
where means the entry is omitted and $\omega_{A}(B(f) B(g))=(f, g)+i(A f, g)$. Such states are called quasi-free.

The quasi-free state $\omega_{0}(A \equiv 0)$ is the unique tracial state on $\mathfrak{A}(\mathscr{H})$ and so is invariant under any automorphism [4].

The first lemma answers the question, "When is the convex combination of quasi-free states quasi-free?" The proof is in the appendix.

Lemma 1. Let $A, B, C$ be anti-hermitian operators in the unit ball of $\mathscr{B}(\mathscr{H})$; let $\omega_{A}, \omega_{B}, \omega_{C}$ be the corresponding quasi-free states and let $0<\lambda<1$. Then,

$$
\omega_{C}=\lambda \omega_{A}+(1-\lambda) \omega_{B}
$$

if and only if $C=\lambda A+(1-\lambda) B$ and there exists an orthonormal pair $\{f, g\}$ and $\mu>0$ such that

$$
A-B=\mu(f \otimes g-g \otimes f) .
$$

If $\{f, g\}$ is an $o-n$ pair of vectors in $H$, we shall define the automorphism $\alpha_{\Theta}(f, g)$ as follows.

$$
\alpha_{\Theta}(f, g) S=\exp ((\Theta / 2) B(g) B(f)) S \exp ((\Theta / 2) B(f) B(g)) \quad \forall S \in \mathfrak{U}(H) .
$$

Let $P$ be a 2-dimensional projector on $H$ and let $J$ be a complex structure on $H$ commuting with $P$ (i.e. $\left.J^{2}=-1, J^{+}=-J\right)$. Let $\tilde{P}_{j}$ denote the group of automorphisms generated by $\left\{\alpha_{\Theta}(f, J f) \mid P f=0\right\}$; and let $\mathfrak{A}\left(H, \tilde{P}_{j}\right)$ denote the algebra of $\tilde{P}_{j}$-fixed points of $\mathfrak{U}(H)$.

Lemma 2. Let $P$ and $J$ be as above. If $H$ is infinite dimensional, then $\mathfrak{A}(P H)=$ $\mathfrak{U}\left(H, \tilde{P}_{j}\right)$.

The proof is in the appendix.

Theorem 3a. Let $\mathscr{H}$ be infinite dimensional; and, let $\alpha$ be $a^{*}$-automorphism of $\mathfrak{U}(\mathscr{H})$ which maps the set of all quasi-free states onto itself. Then $\alpha$ is quasi-free.

Proof. Let $\left\{f_{1}, f_{2}\right\}$ be an $o-n$ pair of vectors in $\mathscr{H}$. By $\alpha$-invariance of $\omega_{0}$, Lemma 1 and linearity of the transpose $\hat{\alpha}$ of $\alpha^{-1}$, we have

$$
(1-\lambda) \omega_{0}+\lambda \hat{\alpha} \omega_{f_{1} \otimes f_{2}-f_{2} \otimes f_{1}}=\hat{\alpha} \omega_{\lambda\left(f_{1} \otimes f_{2}-f_{2} \otimes f_{1}\right)} \quad 0<\lambda<1 .
$$

By hypothesis and Lemma 1, $\hat{\alpha} \omega_{f_{1} \otimes f_{2}-f_{2} \otimes f_{1}}=\omega_{\mu\left(g_{1} \otimes g_{2}-g_{2} \otimes g_{1}\right)}$ for some positive $\mu$ and $o-n$ pair $\left\{g_{1}, g_{2}\right\}$. Now, if $\{h, k\}$ is an arbitrary $o-n$ pair and $\eta$ a real number $(|\eta|<1)$, it follows easily that

$$
\omega_{\eta(h \otimes k-k \otimes h)}=(1+i \eta B(h) B(k)) \circ \omega_{0} .
$$

Since $\omega_{0}$ is faithful and invariant, it follows that $\alpha\left(B\left(f_{1}\right) B\left(f_{2}\right)\right)=\mu B\left(g_{1}\right) B\left(g_{2}\right)$. Squaring both sides gives $\mu=1$. Now let $\left\{f_{3}, f_{4}\right\}$ be an $o-n$ pair, pairwise orthogonal to $\left\{f_{1}, f_{2}\right\}$, and let $\left\{g_{3} g_{4}\right\}$ be so that $\alpha\left(B\left(f_{3}\right) B\left(f_{4}\right)\right)=B\left(g_{3}\right) B\left(g_{4}\right)$.

Now

$$
\begin{aligned}
0 & =\omega_{0}\left(B\left(f_{1}\right) B\left(f_{2}\right) B\left(f_{3}\right) B\left(f_{4}\right)\right) \\
& =\omega_{0}\left(B\left(g_{1}\right) B\left(g_{2}\right) B\left(g_{3}\right) B\left(g_{4}\right)\right)=\left(g_{1}, g_{4}\right)\left(g_{2}, g_{3}\right)-\left(g_{1}, g_{3}\right)\left(g_{2}, g_{4}\right) .
\end{aligned}
$$

And,

$$
\alpha\left[B\left(f_{1}\right) B\left(f_{2}\right), B\left(f_{3}\right) B\left(f_{4}\right)\right]=0=\left[B\left(g_{1}\right) B\left(g_{2}\right), B\left(g_{3}\right) B\left(g_{4}\right)\right] .
$$


Combining these two results, we have

$$
\begin{aligned}
& 0=\omega_{0}\left(B\left(\left(g_{2}, g_{3}\right) g_{4}-\left(g_{2}, g_{4}\right) g_{3}\right) B\left(g_{1}\right) .\right. \\
& \left.\left[B\left(g_{1}\right) B\left(g_{2}\right), B\left(g_{3}\right) B\left(g_{4}\right)\right]\right)=2\left\{\left(g_{2}, g_{3}\right)^{2}+\left(g_{2}, g_{4}\right)^{2}\right\} .
\end{aligned}
$$

Similary $\left(g_{1}, g_{3}\right)=\left(g_{1}, g_{4}\right)=0$. Thus, $\left\{g_{1}, g_{2}\right\}$ is pairwise orthogonal to $\left\{g_{3}, g_{4}\right\}$. Since $\alpha^{-1}$ has this property as well,

$$
\left[\alpha\left(B\left(f_{1}\right)\right), B(h) B\left(h^{\prime}\right)\right]=0=\left[\alpha\left(B\left(f_{2}\right)\right), B(h) B\left(h^{\prime}\right)\right]
$$

for all $o-n$ pairs $\left\{h, h^{\prime}\right\}$ which are pairwise orthogonal to $\left\{g_{1}, g_{2}\right\}$. Let $P$ be the projector upon the subspace spanned by $\left\{g_{1}, g_{2}\right\}$ and let $J$ be a complex structure commuting with $P$. Clearly $\alpha B\left(f_{1}\right)$ and $\alpha B\left(f_{2}\right)$ are $\tilde{P}_{j}$-fixed points of $\mathfrak{U}(H)$. By Lemma 2, there exist numbers $\left\{X_{\gamma}^{j}\right\}_{\gamma=1,2}^{j=1, \ldots 4}$ such that

$$
\alpha\left(B\left(f_{\gamma}\right)\right)=X_{\gamma}^{1} B\left(g_{1}\right)+X_{\gamma}^{2} B\left(g_{2}\right)+X_{\gamma}^{3} B\left(g_{1}\right) B\left(g_{2}\right)+X_{\gamma}^{4} .
$$

Clearly $\omega_{0}\left(\alpha B\left(f_{\gamma}\right)\right)=\omega_{0}\left(\alpha B\left(f_{\gamma}\right) B\left(g_{1}\right) B\left(g_{2}\right)\right)=0$.

Hence $X_{\gamma}^{3}=X_{\gamma}^{4}=0$. It follows from self-adjointness that $X_{\gamma}^{1}$ and $X_{\gamma}^{2}$ are real and from (1) that

$$
\begin{aligned}
& \alpha B\left(f_{1}\right)=B\left(X_{1} g_{1}+X_{2} g_{2}\right) \\
& \alpha B\left(f_{2}\right)=B\left(-X_{2} g_{1}+X_{1} g_{2}\right)
\end{aligned}
$$

with $X_{1}^{2}+X_{2}^{2}=1$.

Now let $\left\{f_{j}\right\}_{j \in \mathbb{Z}}$ be an $o-n$ basis of $H$, and let $\left\{\mathcal{O} f_{j}\right\}$ be the orthonormal basis such that

$$
\alpha B\left(f_{j}\right)=B\left(\mathcal{O} f_{j}\right) .
$$

Let $\mathcal{O}$ be the unique linear, continuous extension of the basis transformation. $\mathcal{O}$ is orthogonal and, by linearity and by continuity,

$$
\alpha B(f)=B(\mathcal{O}) \quad \forall f \in H .
$$

Theorem 3b. Let $H$ be finite dimensional $(\operatorname{dim} H>2)$. There exists $a^{*}$-automorphism of $\mathfrak{A}(H)$ which transforms the set of quasi-free states onto itself and which is not quasi-free.

Proof. It suffices to exhibit a $*$-automorphism $\alpha$ which is non-free, which is trivial on even monomials and commutes with $\alpha_{-1}$. Let $\left\{f_{j}\right\}_{j=1}^{2 N}$ be an orthonormal basis of $H$, let

$$
g_{N}=\left\{\begin{array}{ll}
1 & N \text { odd } \\
i & N \text { even }
\end{array}\right\} \text { and let }
$$

$U=2^{-\frac{1}{2}}\left(1-g_{N} B\left(f_{1}\right) \ldots B\left(f_{2 N}\right)\right) . U$ is unitary and defines an automorphism with the above properties.

$$
U B\left(f_{k}\right) U^{*}=g_{N} B\left(f_{k}\right)\left\{B\left(f_{1}\right) \ldots B\left(f_{2 N}\right)\right\} \quad \forall k .
$$

We now indicate a simpler, alternative proof of Theorem 3.1 of [1]. Let $J$ be a complex-structure on $H$, let $H^{J}$ denote the $J$-complexification of $H$ and let $C$ denote a complex-conjugation on $H^{J}\left(C^{2}=1, C J=-J C, C^{+}=C\right)$. 
A quasi-free state $\omega_{A}$ is said to be gauge invariant if $[J, A]=0$. It is easily checked that $\mu(f \otimes g-g \otimes f)$ defines a gauge invariant quasi-free state if and only if $\mu(f \otimes g-g \otimes f)= \pm \mu(f \otimes J f-J f \otimes f)$. Thus, if $\alpha$ maps the set of gauge invariant quasi-free states onto itself, there exists, for every $f \in H$ a $g \in H$ such that $\alpha(B(f) B(J f))= \pm B(g) B(J g)$. Now

$$
\|B(g) B(J g)+B(h) B(J h)\|
$$

$\geqq\left|\omega_{J}(B(g) B(J g)+B(h) B(J h))\right|=\|g\|^{2}+\|h\|^{2}$. Further, since the $\operatorname{map} f \rightarrow \alpha(B(f) B(J f))$ is continuous, it follows that the two complementary subsets of $\{f \mid\|f\| \geqq \xi\}=H^{\xi}$

$$
H_{ \pm}^{\xi}=\{f \mid\|f\| \geqq \xi, \alpha(B(f) B(J f))= \pm B(g) B(J g) \text { for some } g \in H\}
$$

are both closed and open. Since $H^{\xi}$ is connected $H_{+}^{\xi}=\emptyset$ or $H^{\xi}$. Since $\xi$ is arbitrary, either one of two cases is possible: For every $f \in H$ there exists $g \in H$ such that $\alpha B(f) B(J f)=B(g) B(J g)$; or, for every $f \in H$ there exists $g \in H$ such that $\alpha_{C} \alpha B(f) B(J f)=B(g) B(J g)$. Consider the first possibility. As above $\alpha B(f)$ is a fixedpoint under the action of the group generated by $\left\{\alpha_{\Theta}(k, J k) \mid\{g, J g\} \perp\{k, J k\}\right\}$. By Lemma 2, $\alpha B(f)$ and $\alpha B(J f)$ are elements of the algebra generated by $\{B(g)$, $B(J g)\}$. It follows as above that there exists an orthogonal transformation $\mathcal{O}$ such that $\alpha(B(f))=B(\mathcal{O} f)$ for all $f \in H$. Further $[\mathcal{O}, J]=0$ implying that $\mathcal{O}$ is unitary on $H^{J}$. In the second case, $B(f)=B(\mathcal{O} f)$ with $\mathcal{O}=C \mathcal{O}^{\prime}$ and $\mathcal{O}^{\prime}$ unitary.

It is a pleasure to acknowledge many useful discussions with P.N.M. Sisson and A. Verbeure. The author is indebted to $\mathrm{H}$. Araki for pointing out a fault in an earlier version of this work.

\section{Appendix}

Proof of Lemma 1. Suppose (3) holds. Clearly $C=\lambda A+(1-\lambda) B$. Let $D=A-B$. Then, for arbitrary $\left\{f_{j}\right\}_{j=1}^{4} \subset H$, we have

$$
\begin{aligned}
0 & =\left(\omega_{\lambda A+(1-\lambda) B}-\left(\lambda \omega_{A}+(1-\lambda) \omega_{B}\right)\right)\left(B\left(f_{1}\right) \ldots B\left(f_{4}\right)\right) \\
& =-\lambda(\lambda-1)\left(f_{1},\left\{\left(D f_{3}, f_{4}\right) D-D f_{4} \otimes D f_{3}+D f_{3} \otimes D f_{4}\right\} f_{2}\right) .
\end{aligned}
$$

Since $f_{1}, f_{2}$ are arbitrary, we have

$$
\left(D f_{3}, f_{4}\right) D=D f_{4} \otimes D f_{3}-D f_{3} \otimes D f_{4}
$$

Necessity follows by antisymmetry.

Conversely, suppose that $A-B=\mu(h \otimes g-g \otimes h)$ and $C=\lambda A+(1-\lambda) B$. It is clear that, for $N=2$ or $2 S+1$ ( $S$ a positive integer),

$$
\left(\omega_{\lambda A+(1-\lambda) B}-\left(\lambda \omega_{A}+(1-\lambda) \omega_{B}\right)\right)\left(B\left(f_{1}\right) \ldots B\left(f_{N}\right)\right)=0 .
$$

To prove $\left({ }^{*}\right)$ in general it suffices, by the anticommutation relations (1) to choose $\left\{f_{j}\right\}$ from the elements of an orthonormal basis $\left\{k_{i}\right\}$ with $k_{1}=g, k_{2}=h$ and no entries repeated. We now proceed by induction: Suppose (*) holds for $N=2 S$. By (2) and the induction hypothesis, we have

$$
\begin{aligned}
\left(\omega_{\lambda A+(1-\lambda) B}-\right. & \left(\lambda \omega_{A}+(1-\lambda) \omega_{B}\right)\left(B\left(f_{1}\right) \ldots B\left(f_{2(S+1)}\right)\right) \\
= & i \mu \lambda(1-\lambda) \sum_{j=2}^{2(S+1)}(-1)^{j}\left[\left(g, f_{j}\right)\left(h, f_{1}\right)-\left(f_{1}, g\right)\left(f_{j}, h\right)\right] . \\
& \left(\omega_{A}-\omega_{B}\right)\left(B\left(f_{2}\right) \ldots B\left(f_{j}\right) \ldots B\left(f_{2(S+1)}\right)\right) .
\end{aligned}
$$


We can assume $S \geqq 1$ and anti-commute on the left until $f_{1} \neq g$ and $f_{1} \neq h$, concluding that $(*)$ holds for $N=2(S+1)$.

Q.E.D.

Proof of Lemma 2. Let $C$ denote a complex-conjugation on $H^{J}$ commuting with $P\left(C^{2}=1, C^{+}=C\right.$ and $\left.C J=-J C\right)$. Let $\left\{f_{x}\right\}_{x \in \mathbb{Z}}$ be an orthonormal basis of $(1-P)\left(\frac{1+C}{2}\right) H$ and let $f$ be a normalized vector in $P\left(\frac{1+C}{2}\right) H \cdot \mathfrak{A}((1-P) H)$ is the closed linear span of $\mathfrak{F}_{0} \cup\{I\}$ where

$$
\mathfrak{U}_{0} \equiv\left\{B\left(g_{1}\right) \ldots B\left(g_{N}\right) \mid\left\{g_{n}\right\}_{n=1}^{N} \subset\left\{f_{x}, J f_{x}\right\}_{x \in \mathbb{Z}}\right\}
$$

and $\mathfrak{A}(H)$ is the closed linear span of the set $\mathfrak{U}_{1}$ of elements of the type

$$
S=B(f) S_{1}+B(J f) S_{2}+B(f) B(J f) S_{3}+S_{4}
$$

where $\left\{S_{i}\right\}_{i=1}^{4} \subset \mathfrak{U}_{0}$.

Let

$$
M=\frac{\alpha_{1+P(C-1)}+\alpha_{1}}{2} \cdot \frac{\alpha_{1-2 P}+\alpha_{1}}{2} .
$$

For $S \in \mathfrak{U}_{1}$, we clearly have

$$
S=M(S)+B(f) M(B(f) S)+B(J f) M(B(J f) S)+B(f) B(J f) M(B(J f) B(f) S) .
$$

Since $M$ is linear and continuous this identity holds for all $S \in \mathfrak{A}(H)$. Moreover $M \mathfrak{U}(H)=\mathfrak{A}((1-P) H)$.

It is clear that $S \in \mathfrak{U}\left(H, \tilde{P}_{J}\right)$ iff its coefficients in $\mathfrak{U}((1-P) H)$ are $\tilde{P}_{J}$-fixed points.

Thus, it is sufficient to prove that

$$
\mathfrak{U}((1-P) H) \cap \mathfrak{U}\left(H, \tilde{P}_{J}\right)=\{\mathbb{C} I\} .
$$

To this end define the orthogonal shift $T$ on $H$ by $T f_{x}=f_{x+1}$ and $T J f_{x}=J f_{x+1}$, $T P=P$. It is clear that for all $A \in \mathfrak{U}_{1}, \alpha_{T} A=\lim _{L \rightarrow \infty} \alpha_{T_{L}} A$ where $\left[T_{L}, J\right]=0, P T_{L}=P$
and

$$
T_{L} f_{x}= \begin{cases}f_{x+1} & x \in[-L, L-1] \\ f_{-L} & x=L \\ f_{x} & x \notin[-L, L] .\end{cases}
$$

By linearity and continuity $\alpha_{T}=\lim _{L \rightarrow \infty} \alpha_{T_{L}}$, pointwise. By standard Fourier methods one sees that

$$
\begin{aligned}
\alpha_{T_{L}} & =\prod_{n=1}^{2 L+1} \alpha_{\frac{2 \pi n}{2 L+1}}\left(k_{n}, J k_{n}\right) \quad \text { where } \\
k_{n} & =\frac{1}{\sqrt{2 L+1}} \sum_{x=-L}^{L} e^{-2 \pi J(x+L+1) n / 2 L+1} f_{x} .
\end{aligned}
$$

Thus, if $S \in \mathfrak{U}((1-P) H) \cap \mathfrak{U}\left(H, \tilde{P}_{J}\right)$, then $\alpha_{T} S=S$.

Similarly $\alpha_{2 P-1}=\lim _{L \rightarrow \infty} \prod_{x=-L}^{L} \alpha_{\pi}\left(f_{x}, J f_{x}\right)$, so $\alpha_{2 P-1} S=S$. Now the algebra of $\alpha_{2 P-1}$-fixed points of $\mathfrak{U}((1-P) H)$ is the range of the projector $M \cdot \frac{\alpha_{1}+\alpha_{2 P-1}}{2}$ and so it is the closed span $\mathfrak{U}_{2}$ of the even monomials in $\mathfrak{U}_{0}$. 
Let $S$ be an $\mathfrak{A}\left(H, \tilde{P}_{J}\right)$-fixed point of $\mathfrak{A}((1-P) H)$ and let $S_{n}$ be a Cauchy sequence in $\mathfrak{I}_{2}$ converging to $S$. Now, for each $n$ and $B \in \mathfrak{A}_{1}, \lim _{m \rightarrow \infty}\left\|\left[\alpha_{T^{m}} S_{n}, B\right]\right\|=0$. By linearity, and a $3-\varepsilon$ argument,

$$
\lim _{m \rightarrow \infty}\left[\alpha_{T^{m}} S, B\right]=[S, B]=0 \quad B \in \mathfrak{U}(H) .
$$

Thus $S=\lambda I$ for $\lambda \in \mathbb{C}$.

\section{References}

1. Hugenholts, N., Kadison, R.: Commun. math. Phys. 43, 181-187 (1975)

2. Hugenholtz, N.: Private Communication

3. Balslev, E., Manuceau, J., Verbeure, A.: Commun. math. Phys. 8, 315-326 (1968)

4. Shale, D., Stinespring, W.F.: Ann. Math. 80, 365-381 (1964)

Communicated by $\mathrm{H}$. Araki

Received May 7, 1975; in revised form June 14, 1975 